\title{
On the two varieties of arsenious acid
}

\section{Bussy}

To cite this article: M. Bussy (1847) On the two varieties of arsenious acid, Philosophical Magazine Series 3, 31:206, 151-152, DOI: 10.1080/14786444708645809

To link to this article: http://dx.doi.org/10.1080/14786444708645809

$$
\text { 册 Published online: } 30 \text { Apr } 2009 .
$$

6 Submit your article to this journal $\pi$

LII Article views: 3

Q View related articles $\sqsubset$ 
With this therefore the test solution was prepared, of such a strength, that each division, by measure in the ordinary alkalimeter tube, should be equivalent to the one-tenth or one-fourth of a grain of pure tannin, and thus the number of divisions used would indicate the proportion of available tannin or substance precipitable by gelatin contained in any specimen. A given weight of the sample under trial was then infused in water, or if necessary the astringent matter extracted by boiling, and the clear liquid precipitated by the test solution until no further deposit occurred.

It was necessary in the course of this operation to test at intervals a portion of the solution under examination, to ascertain the progress of the trial ; and this, from the nature of the precipitate, was attended at first with some little difficulty : paper filters were inadmissible from the quantity of the solution they would absorb, and thus introduce a source of extensive error; subsidence rendered the operation very tedious. The plan I have adopted is as follows:-a piece of glass tubing, about twelve inches in length and about half an inch internal diameter, is selected, and this has a small piece of wet sponge loosely introduced into its lower extremity, and when it is wished to abstract a part of the fluid under investigation for a separate testing, this is immersed a few seconds in the partially precipitated solution; the clear liquid then filters by ascent through the sponge into the tube, and is to be decanted from its other extremity into a test glass; if on adding a drop of the gelatin solution to this a fresh precipitate is caused, the whole is returned to the original bulk, and the process proceeded in, and so on until the operation is perfected; this method of operating is facilitated by conducting the examination in a deep glass. After a few trials the manipulation will be found extremely easy, and in this way considerable accuracy may be arrived at,-From the Proceedings of the Chemical Society.

\section{ON THE TWO VARIETIES OF ARSENIOUS ACID. BY M. BUSSY.}

The author first gives a new process for determining the quantity of arsenious acid. This process is based on the employment of standard reagents. The reagent which he uses is permanganate of potash, which M. Marguerite has already successfully employed for the quantitative determination of iron.

When a solution of permanganate of potash is poured into a solution of arsenious acid, it becomes arsenic acid, and the red colour of the reagent disappears. The liquor begins to become coloured only when the transformation of arsenious acid is complete. When, then, a standard solution of permanganate of potash is prepared, the quantity of arsenious acid contained in any solution may be determined by that of the permanganate required to convert it into arsenic acid.

M. Bussy states that the two varieties of arsenious acid, the vitreous and opake, absorb the same quantity of permunganate, and consequently that the differences observed in their solubility is not derived from any difference of oxidizement.

With respect to the solubility of the two varieties of arsenious 
acid, M. Bussy has arrived at the following conclusions:-lst. The vitreous, so far from being less soluble in water than the opake acid, as stated by chemists, is, on the contrary, much more soluble. This difference is nearly in the proportion of 3 to 1 , at about $53^{\circ}$ to $55^{\circ}$ of F.; the same quantity of water which dissolves 36 to 38 parts of the vitreous acid, will take up only 12 to 14 of the opake. 2nd. The vitreous acid dissolves much more rapidly than the opake acid. 3rd. Neither of the varieties possesses a degree of solubility which is to be regarded as strictly peculiar to it. 4th. The opake acid is converted into vitreous acid by long boiling in water; that is to say, it then acquires the same degree of solubility as the vitreous arsenious acid, which is such that 11 parts are dissolved by 100 of water. 5th. Under the influence of water and a low temperature, the vitreous acid is converted into opake acid; that is to say, a solution of vitreous acid becomes reduced after a certain time to the point of saturation which belongs to the opake acid. 6th. The mixture of the two varieties of acid in the same solution explains the anomalies observed in the solubility of arsenious acid, which in fact offers nothing opposed to the principles admitted by chemists. 7 th. Division, which facilitates the solution of the opake acid, without however increasing its solubility, considerably diminishes that of the vitreous acid; and to such an extent, that this acid, reduced to fine powder and levigated, is not sensibly more soluble in water than the opake acid; this resulting unquestionably from a transformation which it undergoes, either at the moment of pulverization, or of its contact with water. 8 th. Acid which has been rendered opake by the action of ammonia, and acid crystallized in water, act similarly with water, and appear to belong to the samevariety. 9 th. The opake acid dissolves more slowly than the vitreous in dilute hydrochloric acid. This circumstance, which thus modifies the nature of the products formed during solution, explains why the luminous phænomena observed by $M$. Rose in the crystallization of the vitreous acid, are not in general observable with so great intensity in the solution of the opake variety. 10th. The difference which has been observed in the action of the two arsenious acids on tincture of litmus is merely apparent. If the opake acid does not redden the tincture, it is on account of its slight solubility, and especially because it dissolves slowly; whilst the vitreous acid, which dissolves quickly, immediately reddens the tincture. But if comparative experiments be made, and the tincture be exposed to the action of the powder, it becomes gradually red, and no difference is perceptible at the expiration of three or four days.-Comptes Rendus, Mai 1847.

\section{ON THE PREPARATION OF GUN-COTTON.}

Mr. Coathupe recently forwarded to the Chemical Society two specimens of gun-cotton, with a view to illustrate the greatly increased explosive effects that are to be derived from a subsequent immersion of the gun-cotton, when properly prepared in the ordi. nary way, in a saturated solution of chlorate of potash. 